Contributions to Achenium Leach, 1819 (Coleoptera, Staphylinidae, Paederinae) fauna of Turkey

İnanç AYAN ${ }^{1}$, Sinan ANLAŞ²

${ }^{1}$ Alaşehir Municipality, Alaşehir, Manisa, Turkey. ${ }^{2}$ Manisa Celal Bayar University, Alaşehir Vocational School, Manisa, Turkey. ${ }^{1}$ https://orcid.org/0000-0003-0339-2946, ${ }^{2}$ https://orcid.org/0000-0001-7059-5677,

$\bowtie$ : sinan.anlas@gmail.com

\section{ABSTRACT}

In this study, total of 388 specimens of the genus Achenium Leach, 1819 (Coleoptera: Staphylinidae) were collected by aspirator and sifter methods from different provinces of Aegean and Central Anatolia Regions of Turkey between 2014 and 2019. As a result of the field studies, five species of the genus were recorded. These species wereAchenium anatolicum Jarrige, 1952; A. humile (Nicolai, 1822); A. propontiacum Bordoni, 2009; A. scimbalioides Koch, 1937 and $A$. turcicum Coiffait, 1971. Of the recorded species; $A$. humile from the Aegean Region and $A$. turcicum from Central Anatolia Region were the first recorded for the area. A. turcicum was reported for the first time outside the type locality and its distribution was found to be much wider than previously known. A. turcicum and $A$. anatolicum were the endemic species for Anatolia.

\section{Research Article}

\section{Article History}

Received

$: 11.02 .2020$

Accepted : :08.05.2020

\section{Keywords}

Paederinae

Achenium

Fauna

Endemism

Turkey

\title{
Türkiye Achenium Leach, 1819 (Coleoptera, Staphylinidae, Paederinae) Faunasına Katkılar
}

\section{ÖZET}

$\mathrm{Bu}$ çalışmada, 2014-2019 yılları arasında aspiratör ve eleme yöntemleri ile Ege ve İç Anadolu Bölgelerinden Achenium Leach, 1819 (Coleoptera: Staphylinidae) cinsine bağlı toplam 388 örnek toplanmıştır. Arazi çalışmaları sonucunda beş tür kaydedilmiştir. Bunlar Achenium anatolicum Jarrige, 1952; A. humile (Nicolai, 1822); A. propontiacum Bordoni, 2009; A. scimbalioides Koch, 1937 and $A$. turcicum Coiffait, 1971 türleridir. Kaydedilen türlerden, $A$. humile türü Ege Bölgesi’nden ve $A$. turcicum türü de İç Anadolu Bölgesi'nden ilk defa bildirilmiştir. A. turcicum türü tip lokalitesi dışından ilk kez rapor edilmiş olup yayılışının bilinenden çok daha geniş olduğu bulunmuştur. Ayrica, A. turcicum ve A. anatolicum türleri, Anadolu endemiği türlerdir.

\section{Araştırma Makalesi}

\author{
Makale Tarihçesi \\ Geliş Tarihi : 11.02 .2020 \\ Kabul Tarihi : 08.05 .2020
}

\section{Anahtar Kelimeler}

Paederinae

Achenium

Fauna

Endemizm

Türkiye

To Cite : Ayan I, Anlaş S 2020. Contributions to Achenium Leach, 1819 (Coleoptera, Staphylinidae, Paederinae) fauna of Turkey. KSU J. Agric Nat 23 (4): 904-912. DOIः 10.18016/ksutarimdoga.vi.687967.

\section{INTRODUCTION}

According to a recent comprehensive revision by Assing (2010), the genus Achenium Leach, 1819 (Staphylinidae, Paederinae) contains 53 species worldwide. In the meantime, a new species were described from Russia (Solodovnikov \& Li, 2013). The biodiversity center of Achenium is the Mediterranean countries. Up to date, 11 species of the genus Achenium were recorded from Turkey, two of them are known from only this country (Assing, 2010; Anlaş et al., 2011; Schülke \& Smetana, 2015).

Despite many recent contributions, the current knowledge of the Achenium fauna of Turkey is far from complete. The main purpose of this study was to make a contribution to fauna of Achenium of Turkey.

\section{MATERIAL and METHODS}

The material has been obtained by aspirator and sifter methods in different field studies between 20142019 in Aegean and Central Anatolia Regions. Specimens were examined using a Stemi 508 microscope (Zeiss, Oberkochen, Germany). Photographs of the habitus, forebody and aedeagus were taken with a digital camera (Zeiss Axiocam ERC5s) All photographs were edited with the Helicon Focus v. 6, and Corel Draw v. X5 software.

The material referred to in the paper was stored in the collection of the Alaşehir Zoological Museum, of Manisa Celal Bayar University (AZMM). Classification and nomenclature of the genus Achenium suggested by Assing (2010) and Schülke and Smetana (2015) have been followed in the study. 


\section{RESULTS}

In this study, five species of the genus Achenium belonging to the subfamily Paederinae were reported from Aegean and Central Anatolia regions of Turkey. Amongst them, A. humile from the Aegean Region and $A$. turcicum from Central Anatolia Region were recorded for the first time. A. turcicum was reported for the first time outside the type locality and its distribution was found to be much wider than previously known.

\section{Achenium anatolicum Jarrige, 1952 (Figure 1)}

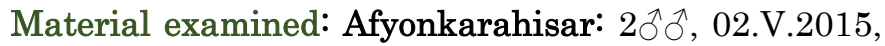
Ahır Mountains, Büyükhacet Hill, $38^{\circ} 39^{\prime} 42^{\prime \prime N}$, $30^{\circ} 06^{\prime} 05 " \mathrm{E}, 1925 \mathrm{~m}$, leg. Yağmur \& Örgel. Aksaray: $8 \widehat{\partial^{\circ}}$, 8우우, 21.III.2018, Güzelyurt, Şahinkalesi Hill, $38^{\circ} 17^{\prime} 16^{\prime \prime} \mathrm{N}, 34^{\circ} 25^{\prime} 50^{\prime \prime} \mathrm{E}, 1910 \mathrm{~m}$, leg. Örgel \& Yaman;

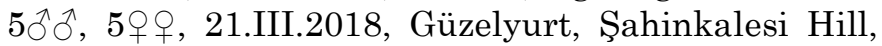
$38^{\circ} 16^{\prime} 16^{\prime \prime} \mathrm{N}, 34^{\circ} 24^{\prime} 37^{\prime \prime E}, 1860 \mathrm{~m}$, leg. Örgel \& Yaman. Denizli: $3 \widehat{\widehat{\partial},}$ 24.IV.2014, Honaz Mountain, $37^{\circ} 39^{\prime} 50^{\prime \prime N}, 29^{\circ} 17^{\prime} 36^{\prime \prime E}, 2077 \mathrm{~m}$, leg. Anlaş \& Örgel.

Karaman: 1ㅅ, 3웅, 25.V.2016, Sariveliler, Göktepe, Saçak Hill, 36³ $38^{\prime} 22^{\prime \prime N}, 32^{\circ} 32^{\prime} 09^{\prime \prime E}, 1847 \mathrm{~m}$, leg. Örgel \& Yaman. Kayseri: $2 \hat{\jmath} \hat{\jmath}, 2$ 우, 08.IV.2018,

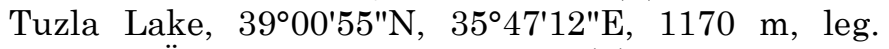

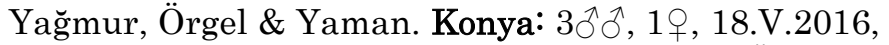
Beyşehir Lake, $37^{\circ} 35^{\prime} 54^{\prime \prime} \mathrm{N}, 31^{\circ} 26^{\prime} 41^{\prime \prime} \mathrm{E}$, leg. Örgel \& Yaman; 2ภㅅ․ 3웅, 24.V.2016, Sariveliler $25 \mathrm{~km} \mathrm{W,}$ $36^{\circ} 52^{\prime} 08^{\prime \prime N}$, 32²5'19"E, 1976 m, leg. Örgel \& Yaman;

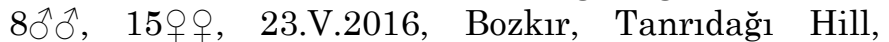
$36^{\circ} 56^{\prime} 29^{\prime \prime} \mathrm{N}, 32^{\circ} 11^{\prime} 12^{\prime \prime E}, 2085 \mathrm{~m}$, leg. Örgel \& Yaman; 2万人스, 4우우, 24.V.2016, Hadim, Beyreli road, $36^{\circ} 53^{\prime} 01 " \mathrm{~N}, 32^{\circ} 25^{\prime} 10^{\prime \prime E}, 1933 \mathrm{~m}$, leg. Örgel \& Yaman;

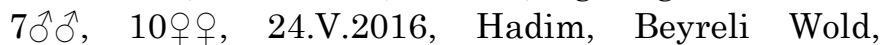
$36^{\circ} 53^{\prime} 06 " \mathrm{~N}, 32^{\circ} 22^{\prime} 31$ "E, $2016 \mathrm{~m}$, leg. Örgel \& Yaman;

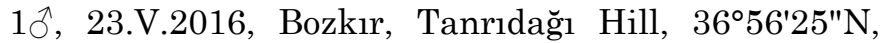
$32^{\circ} 09^{\prime} 18 " \mathrm{E}, 2071 \mathrm{~m}$, leg. Örgel \& Yaman; 10, 1우, 20.V.2016, Seydişehir, Erenler Mountain, 37² $34^{\prime} 40^{\prime N}$,

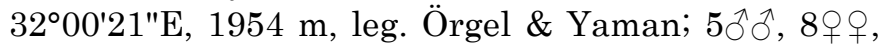
04.III.2018, Karapınar, Yeşilyurt, Ovacık Wold, $37^{\circ} 45^{\prime} 57^{\prime \prime} \mathrm{N}, 33^{\circ} 46^{\prime} 08^{\prime \prime} \mathrm{E}, 1600 \mathrm{~m}$, leg. Örgel \& Yaman; 3새, 5우우, 30.IV.2018, Bozkır, Geyik Mountains, $1875 \mathrm{~m}, 36^{\circ} 58^{\prime} 39^{\prime \prime} \mathrm{N}, 32^{\circ} 04^{\prime} 56^{\prime \prime} \mathrm{E}$, leg. Örgel \& Yaman;

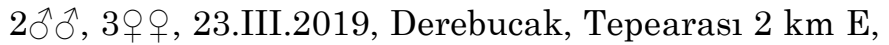
$37^{\circ} 29^{\prime} 23^{\prime \prime N}$, 31 $31^{\circ} 40^{\prime} 47^{\prime \prime E}, 1530 \mathrm{~m}$, leg. Örgel \& Köksal. Kütahya: 1ิे, 03.V.2015, Murat Mountain, $38^{\circ} 56^{\prime} 15^{\prime \prime} \mathrm{N}, 2^{\circ} 35^{\prime} 45^{\prime \prime} \mathrm{E}, 1754 \mathrm{~m}$, leg. Yağmur \& Örgel; 3ภ̂े, 2우, 03.V.2015, Murat Mountain, $38^{\circ} 55^{\prime} 04^{\prime \prime N}, 29^{\circ} 36^{\prime} 47^{\prime \prime E}, 1756 \mathrm{~m}$, leg. Yağmur \& Örgel. Nevşehir: 10̂, 26.III.2018, Hacıbektaş, $38^{\circ} 56^{\prime} 22^{\prime \prime} \mathrm{N}, 34^{\circ} 37^{\prime} 40^{\prime \prime} \mathrm{E}, 1210 \mathrm{~m}$, leg. Örgel \& Yaman.

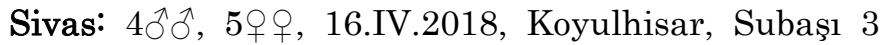
$\mathrm{km}$ E, $40^{\circ} 20^{\prime} 39^{\prime \prime} \mathrm{N}, 37^{\circ} 49^{\prime} 37^{\prime \prime E}, 1790 \mathrm{~m}$, leg. Anlaş \&

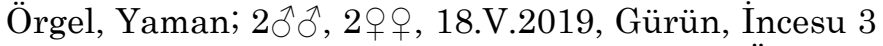
$\mathrm{km} \mathrm{E}, 38^{\circ} 45^{\prime} 17^{\prime \prime} \mathrm{N}, 37^{\circ} 08^{\prime} 49^{\prime \prime} \mathrm{E}, 1690 \mathrm{~m}$, leg. Örgel \&

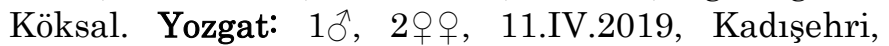
Ovacık 4 km N, 4004'58"N, 3553'58"E, $1670 \mathrm{~m}$, leg.
Yağmur \& Örgel, Yaman. Totally: 136 specimens.

Distribution in Turkey: A. anatolicum was known from Ankara, Antalya, Bayburt, Burdur, Çanakkale, Çankırı, Corum, Eskişehir, Isparta, İzmir, Karaman, Kırşehir, Konya, Kütahya, Manisa, Muğla and Uşak (Jarrige, 1952; Anlaş, 2009; Assing, 2010, 2013; Anlaş et al., 2011; Örgel \& Anlaş, 2016). This species is recorded from Afyonkarahisar, Aksaray, Denizli, Kayseri, Nevşehir ve Sivas provinces for the first time.

Distribution in the world: This species only occurs in Turkey (Schülke \& Smetana, 2015). Endemic.

\section{Achenium humile (Nicolai, 1822) (Figure 2)}

Material examined: Afyonkarahisar: 10ิ, 02.V.2015, Ahır Mountains, Büyükkavşak Hill, $38^{\circ} 43^{\prime} 08^{\prime \prime N}$, $30^{\circ} 03^{\prime} 48^{\prime \prime} \mathrm{E}, 1810 \mathrm{~m}$, leg. Yağmur \& Örgel. Aksaray: 10ิ, 1ㅇ, 23.III.2018, Ekecik Mountain, Radar road, $38^{\circ} 38^{\prime} 36^{\prime \prime} \mathrm{N}, 34^{\circ} 01^{\prime} 30 " \mathrm{E}, 1700 \mathrm{~m}$, leg. Örgel \& Yaman; $7 \widehat{\partial}^{\circ}$, 10우우, 21.III.2018, Güzelyurt, Şahinkalesi, Hill, $38^{\circ} 17^{\prime} 16^{\prime \prime} \mathrm{N}, 34^{\circ} 25^{\prime} 50^{\prime \prime} \mathrm{E}, 1910 \mathrm{~m}$, leg. Örgel \& Yaman;

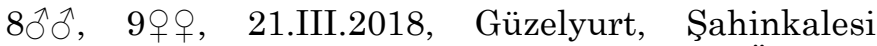

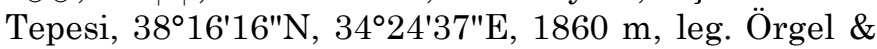
Yaman. Ankara: $3 \hat{0}^{\hat{\alpha}}$, 6우, 18.V.2018, Kızılcahamam, Yukarıçanlı $8 \mathrm{~km}$ N, $1650 \mathrm{~m}$, $40^{\circ} 43^{\prime} 43^{\prime \prime N}, 32^{\circ} 41^{\prime} 15 " \mathrm{E}$, leg. Örgel \& Yaman. Çankırı: 10ิ, 21.V.2018, Ilgaz, Ilgaz Mountains, $1860 \mathrm{~m}$,

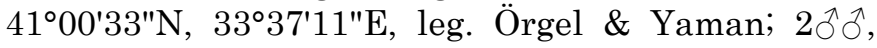
7우우, 20.V.2018, Kurşunlu $5 \mathrm{~km} \mathrm{E,} \mathrm{Taşkaracalar,}$ $1647 \mathrm{~m}, 40^{\circ} 42^{\prime} 11^{\prime \prime N}, 33^{\circ} 19^{\prime} 43^{\prime} \mathrm{E}$, leg. Örgel \& Yaman; 10ิ, 1ㅇ, 19.V.2018, Atkaracalar, Ilipmar $5 \mathrm{~km} \mathrm{SE}$, $1750 \mathrm{~m}, 40^{\circ} 46^{\prime} 10^{\prime \prime} \mathrm{N}, 33^{\circ} 09^{\prime} 32^{\prime \prime} \mathrm{E}$, leg. Örgel \& Yaman; 10, 4우우,20.V.2018, Korgun, Çukurören $3 \mathrm{~km}$ SW, $1390 \mathrm{~m}, 40^{\circ} 38^{\prime} 55^{\prime \prime} \mathrm{N}, 33^{\circ} 22^{\prime} 05^{\prime \prime} \mathrm{E}$, leg. Örgel \& Yaman; 10ิ, 1ㅇ, 21.V.2018, Ilgaz, Kuyupinar $2 \mathrm{~km}$ E, $1414 \mathrm{~m}$, $40^{\circ} 51^{\prime} 06^{\prime \prime N}, 33^{\circ} 37^{\prime} 37^{\prime \prime E}$, leg. Örgel \& Yaman. Eskişehir: 10ิ, 27.III.2018, Türkmen Mountain, $39^{\circ} 26^{\prime} 22^{\prime \prime} \mathrm{N}, 30^{\circ} 22^{\prime} 22^{\prime \prime} \mathrm{E}, 1660 \mathrm{~m}$, leg. Örgel \& Yaman. Kayseri: $3 \hat{\partial} \hat{\partial}, 3$ 우우 09.IV.2018, Pınarbaşı, Malakköy $2 \mathrm{~km}$ SW, Hinzır Mountains, 3902'33"N, 36²0'47"E,

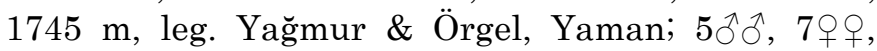
09.VI.2016, Pınarbaşı, Eşkiyassıpınar, Gövdeli

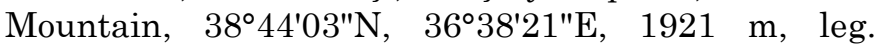
Yağmur, Örgel \& Yaman; 10, 1ㅇ, 09.VI.2016, Pınarbaşı, Yağlıpınar, 3849'10"N, 36²2'17"E, leg.

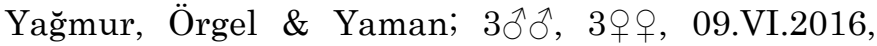

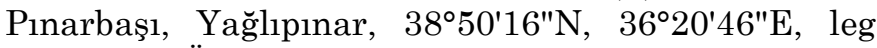
Yağmur, Örgel \& Yaman. Kütahya: 1, 10, 10.V.2015, Şaphane Mountain, $39^{\circ} 02^{\prime} 48^{\prime \prime} \mathrm{N}$, $29^{\circ} 18^{\prime} 17 " \mathrm{E}, 1658 \mathrm{~m}$, leg. Yağmur \& Örgel. Niğde: $1 \mathrm{o}^{\prime}$, 2ㅇ, 03.VI.2016, Çamardı, Demirkazık, $2259 \mathrm{~m}$, leg. Örgel \& Yaman. Sivas: 10̂, 18.IV.2018, İmranll, Yürektaşı $2 \mathrm{~km}$ güney, 39 $35^{\prime} 31^{\prime \prime N}, 38^{\circ} 15^{\prime} 50^{\prime \prime} \mathrm{E}, 1420$ $\mathrm{m}$, leg. Anlaş \& Örgel, Yaman; 2へ̂̀, 13.IV.2019, Ylldız Mountains ski resort, $40^{\circ} 08^{\prime} 18^{\prime \prime} \mathrm{N}, 36^{\circ} 56^{\prime} 18^{\prime \prime} \mathrm{E}$, 
1730 m, leg. Anlaş, Örgel \& Köksal; 2へ̂̄, 3ㅇ, 16.IV.2019, İmranlı, Sivas-Erzincan road, Kızıldağ Pass, 3951'19"N, 38²4'57"E, 2125 m, leg. Anlaş, Örgel \& Köksal. Yozgat: $4 \overbrace{}^{\hat{\partial}}$, 2우, 11.IV.2019,
Kadışehri, Ovacık $4 \mathrm{~km} \mathrm{~N}, 40^{\circ} 04^{\prime} 58^{\prime \prime N}, 35^{\circ} 53^{\prime} 58^{\prime \prime} \mathrm{E}$, 1670 m, leg. Yağmur \& Örgel, Yaman. Totally: 110 specimens.

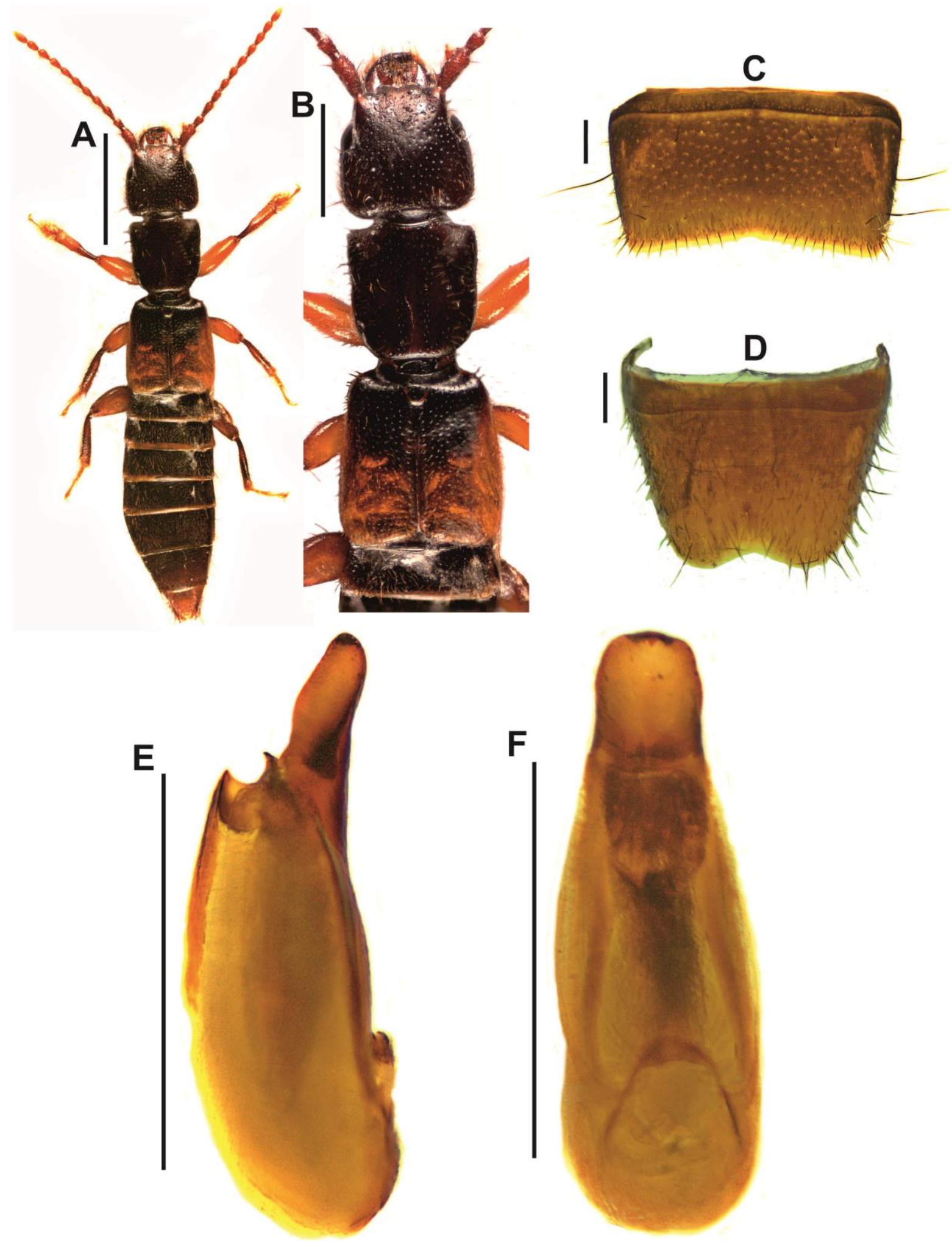

Figure 1. Achenium anatolicum Jarrige. A-habitus; B-forebody; C-male sternite VII; D-male sternite VIII; E-aedeagus, lateral; F-aedeagus, ventral. Scale bars: $1 \mathrm{~mm}(\mathrm{~A}-\mathrm{B}) ; 0.2 \mathrm{~mm}(\mathrm{C}-\mathrm{D}) ; 0.5 \mathrm{~mm}(\mathrm{E}-\mathrm{F})$

Şekil 1. Achenium anatolicum Jarrige. A-genel vücut; B-ön vücut; C-erkek sternit VII; D-erkek sternit VIII; $E$ aedeagus, yan görünüş; F-aedeagus, ön görünüş. Ölçek çubukları: $1 \mathrm{~mm}(A-B) ; 0.2 \mathrm{~mm}(C-D) ; 0.5 \mathrm{~mm}(E-F)$. 

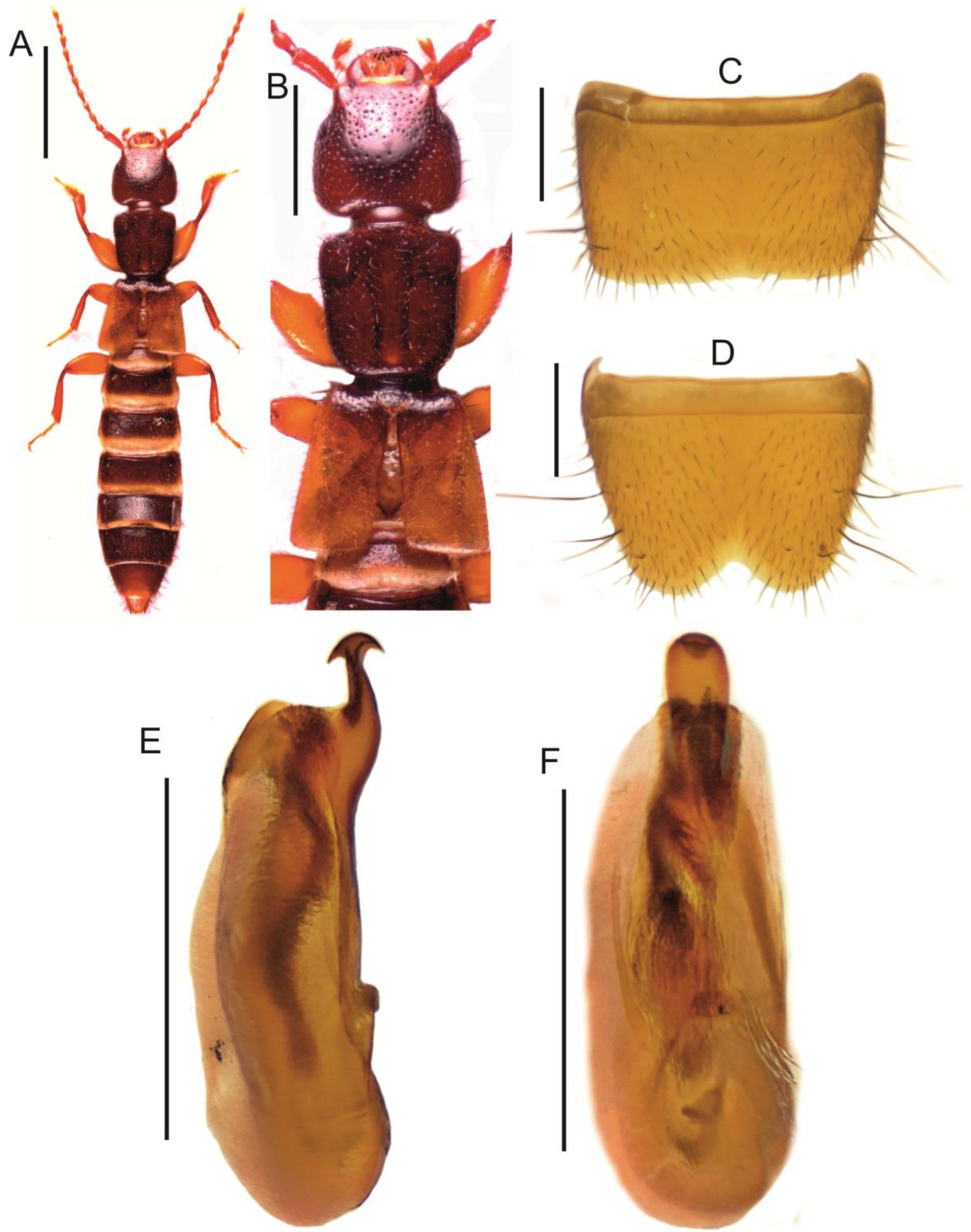

Figure 2. Achenium humile (Nikolai). A-habitus; B-forebody; C-male sternite VII; D-male sternite VIII; E-aedeagus, lateral; F-aedeagus, ventral. Scale bars: $1 \mathrm{~mm}$ (A-B); $0.2 \mathrm{~mm}(\mathrm{C}-\mathrm{D}) ; 0.5 \mathrm{~mm}(\mathrm{E}-\mathrm{F})$.

Şekil 2. Achenium humile (Nikolai). A-genel vücut; B-ön vücut; $C$-erkek sternit VII; $D$-erkek sternit VIII; E-aedeagus, yan görünüş; F-aedeagus, ön görünüş. Ölçek çubukları: $1 \mathrm{~mm}(A-B) ; 0.2 \mathrm{~mm}(C-D) ; 0.5 \mathrm{~mm}(E-F)$.

Distribution in Turkey: Ankara, Erzincan, Eskişehir, Gümüşhane, Isparta, Kars, Kastamonu, Muş, Niğde, Tunceli, Van (Anlaş, 2009; Anlaş et al., 2011; Assing 2010, Sert et al., 2013; Altın \& Yağmur, 2018; Özgen et al., 2018). A. humile is recorded for the first time from Aegean Region of Turkey.

Distribution in the world: This species is widely distributed in Europe and also known from Middle Asia and Turkey (Schülke \& Smetana, 2015).

Achenium propontiacum Bordoni, 2009 (Figure 3)

Material examined: Ankara: $3 \widehat{\jmath} \hat{\jmath}, 5$ 우, 11.IV.2017, Beypazarı, Üreğil, 40¹7'07"N, 3204'11"E, $1375 \mathrm{~m}$, leg. Örgel \& Yaman; 1ㅇ, 25.IX.2017, Beypazarı, 


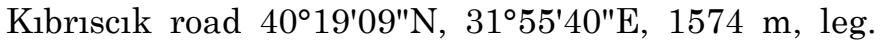
Örgel \& Yaman; 10, 10.III.2018, Mamak,

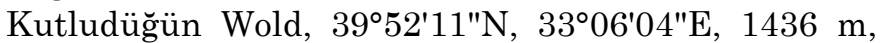

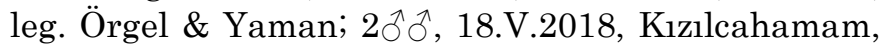
Yukarıçanlı $8 \mathrm{~km} \mathrm{~N}, 1650 \mathrm{~m}, 40^{\circ} 43^{\prime} 43^{\prime \prime} \mathrm{N}, 32^{\circ} 41^{\prime} 15^{\prime \prime} \mathrm{E}$,

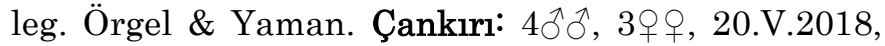
Kurşunlu, Taşkaracalar $2 \mathrm{~km} \mathrm{E,} 1510 \mathrm{~m}, 40^{\circ} 42^{\prime} 40^{\prime \prime} \mathrm{N}$, $33^{\circ} 17^{\prime} 21 " \mathrm{E}$, leg. Örgel \& Yaman; 10, 2 우, 20.V.2018, Korgun, Çukurören $3 \mathrm{~km} \mathrm{SW}, 1390 \mathrm{~m}, 40^{\circ} 38^{\prime} 55^{\prime \prime} \mathrm{N}$, $33^{\circ} 22^{\prime} 05 " \mathrm{E}$, leg. Örgel \& Yaman; $1 \jmath^{\hat{\prime}}, 2$ 우,, $21 . V .2018$, Ilgaz, Kuyupinar $2 \mathrm{~km} \mathrm{E,} 1414 \mathrm{~m}, 40^{\circ} 51^{\prime} 06^{\prime \prime N}$,

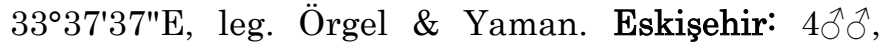
5 우우, 27.IV.2019, Sarıcakaya, İğdir $8 \mathrm{~km} \mathrm{~N}$, $40^{\circ} 07^{\prime} 10^{\prime \prime N}, 30^{\circ} 36^{\prime} 47^{\prime \prime E}, 1200$ m, leg. Örgel \& Köksal; 10ิ, 1ㅇ, 27.IV.2019, Sarıcakaya, İğdir $6 \mathrm{~km} \mathrm{S,}$ $40^{\circ} 06^{\prime} 08^{\prime \prime N}$, 30³7'02"E, 1200 m, leg. Örgel \& Köksal. Totally: 36 specimens.

Distribution in Turkey: Ankara, Ballkesir, Edirne, Erzurum, Istanbul, Kastamonu, Kırklareli, Kocaeli, Samsun, Sinop, Tekirdağ (Bordoni, 2009; Anlaş et al., 2011; Assing 2010). This species is here recorded for the first time from Çankırı and Eskişehir provinces.

Distribution in the world: This species is distributed in Bulgaria, Greece, Moldova, Romania, Turkey and Ukraine (Schülke \& Smetana, 2015).

\section{Achenium scimbalioides Koch, 1937 (Figure 4)}

Material examined: Afyonkarahisar: 10, 01.VI.2014,

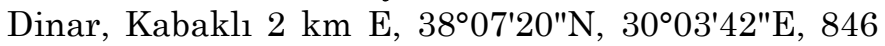
$\mathrm{m}$, leg. Yağmur \& Örgel. Aksaray: 1ㅇ, 22.III.2018, Melendiz River, $38^{\circ} 12^{\prime} 00^{\prime \prime} \mathrm{N}, 34^{\circ} 19^{\prime} 50^{\prime \prime} \mathrm{E}, 1400 \mathrm{~m}$, leg. Örgel \& Yaman. Ankara: 10, 09.IV.2017, Beypazarı, Üreğil, $40^{\circ} 17^{\prime} 07^{\prime \prime N}, 32^{\circ} 04^{\prime} 11^{\prime \prime} \mathrm{E}, 1375 \mathrm{~m}$, leg. Örgel \& Yaman. Aydın: 2ภํ, 22.III.2015, Dilek Peninsula

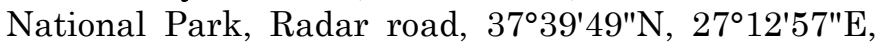
969 m, leg. Yağmur \& Örgel. Çankırı: 10̂, 20.V.2018, Kurşunlu 5 km E, Taşkaracalar, 1647 m, 40² $42^{\prime} 11^{\prime \prime N}$,

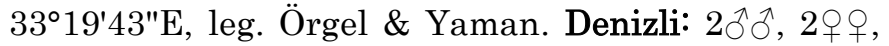
11.IV.2015, Tavas, 3739'36"N, 28 52'06"E, $1131 \mathrm{~m}$, leg. Anlaş, Yağmur \& Örgel. Eskişehir: $1 \hat{0}, 2$ 우우, 27.IV.2019, Sarıcakaya, İğdir $8 \mathrm{~km} \mathrm{~N}, 40^{\circ} 07^{\prime} 10^{\prime \prime N}$,

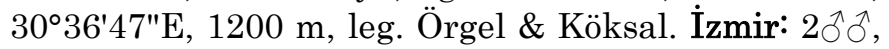
2 우우, 14.XI.2014, Karaburun $5 \mathrm{~km}$ SW, 38 $37^{\circ} 39^{\prime \prime}$, $26^{\circ} 29^{\prime} 26 " \mathrm{E}$, leg. Anlaş, Yağmur \& Örgel. Karaman:

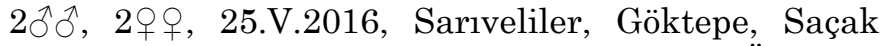
Hill, 36 $38^{\circ} 22^{\prime \prime N}, 32^{\circ} 32^{\prime} 09 " \mathrm{E}, 1847 \mathrm{~m}$, leg. Örgel \& Yaman. Kütahya: $14 \hat{\jmath}$, 8 우, 03.V.2015, Murat Mountain, 38 $55^{\prime} 04^{\prime \prime} \mathrm{N}, 2^{\circ} 36^{\prime} 47^{\prime \prime E}, 1756 \mathrm{~m}$, leg. Yağmur, Örgel \& Altın. Manisa: 5 $\hat{\jmath}, 5$ 우,

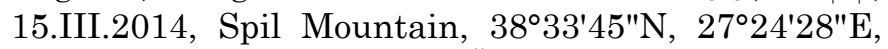

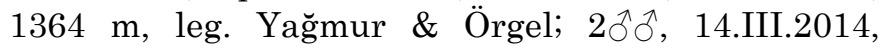
Selendi, Omurlar village, $38^{\circ} 53^{\prime} 04^{\prime \prime N}$, $28^{\circ} 49^{\prime} 27^{\prime \prime E}, 868$

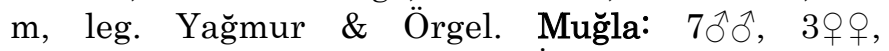
22.III.2014, Ylanlı Orman İşletme $2 \mathrm{~km} \mathrm{~N}$, $37^{\circ} 13^{\prime} 52^{\prime \prime} \mathrm{N}, 28^{\circ} 29^{\prime} 28^{\prime \prime} \mathrm{E}, 1172 \mathrm{~m}$, leg. Anlaş \& Örgel.
Niğde: 1ภ, 1, 02.VI.2016, Ulukışla, Horoz, $37^{\circ} 28^{\prime} 47^{\prime \prime N}$, 34' $47^{\prime} 55 " \mathrm{E}, 1049 \mathrm{~m}$, leg. Örgel \& Yaman. Yozgat: $1 \delta^{\hat{O}}, 4$ 우, 11.IV.2019, Kadışehri, Ovacık $4 \mathrm{~km}$ $\mathrm{N}, 40^{\circ} 04^{\prime} 58^{\prime \prime} \mathrm{N}, 35^{\circ} 53^{\prime} 58^{\prime \prime} \mathrm{E}, 1670 \mathrm{~m}$, leg. Yağmur \& Örgel, Yaman. Totally: 72 specimens.

Distribution in Turkey: Adana, Bayburt, Çanakkale, Gümüşhane, Manisa, Tunceli, Uşak (Anlaş, 2009; Anlaş et al., 2011; Assing 2010, Örgel \& Anlaş, 2016). This species is here recorded for the first time from many provinces (see above) in Aegean and Central Anatolia Regions.

Distribution in the world: Bulgaria, Turkey and Greece (Schülke \& Smetana, 2015).

\section{Achenium turcicum Coiffait, 1971 (Figure 5)}

Material examined: Karaman: 10ิ, 28.V.2016, Ayranc1-Erdemli Yolu, Yüğlük Hill, 3702'49"N, $34^{\circ} 00^{\prime} 26 " \mathrm{E}, 2176 \mathrm{~m}$, leg. Anlaş, Örgel \& Yaman; 10̂,

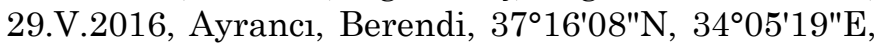
$1832 \mathrm{~m}$, leg. Anlaş, Örgel \& Yaman; 19, 29.V.2016, Ayranc1, Berendi $10 \mathrm{~km} \mathrm{~N}, 37^{\circ} 16^{\prime} 02^{\prime \prime N}, 34^{\circ} 06^{\prime} 53^{\prime \prime} \mathrm{E}$,

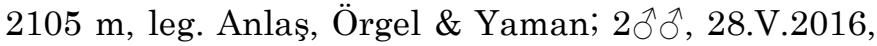

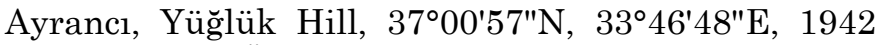
$\mathrm{m}$, leg. Anlaş, Örgel \& Yaman; $8 \hat{0}^{\hat{\gamma}}, 7$ 웅, 03.V.2018,

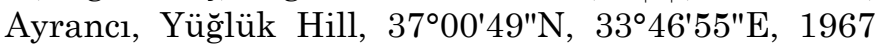

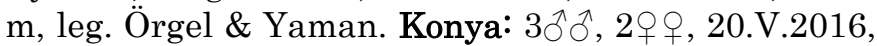
Seydişehir, Erenler Mountain, $37^{\circ} 34^{\prime} 29^{\prime \prime} \mathrm{N}$, $32^{\circ} 02^{\prime} 56 " \mathrm{E}, 1785 \mathrm{~m}$, leg. Örgel \& Yaman; $10^{\wedge}, 5$ 우우, 19.V.2016, Beyşehir, Erenler Mountain, 37²4'20"N, $31^{\circ} 58^{\prime} 31^{\prime \prime E}, 1770 \mathrm{~m}$, leg. Örgel \& Yaman; 10ิ, 1우, 05.III.2018, Böllük Lake, 38³0'49"N, 3254'00"E, 990 m, leg. Örgel \& Yaman; 1ठ, 02.III.2018, Ilgın, Bulcuk, $38^{\circ} 06^{\prime} 52^{\prime \prime N}, 31^{\circ} 59^{\prime} 03 " \mathrm{E}, 1436 \mathrm{~m}$, leg. Örgel \& Yaman. Totally: 34 specimens.

Distribution in Turkey: A. turcicum was only known its type locality, Şarkikaraağaç, Isparta (Coiffait, 1971; Assing, 2010). Thus, this species is recorded for the first time outside the type locality and its distribution is found to be much wider than it was previously known. A. turcicum is recorded for the first time from Central Anatolia Region.

Remarks: For redescription of this species, see Assing (2010). Aedeagus in ventral view and male sternite VII of $A$. turcicum are given for the first time in Figures 5C, F.

Distribution in the world: This is an endemic species for Turkey (Schülke \& Smetana, 2015).

\section{DISSCUSSION}

The genus Achenium Leach includes 54 species in the Palaearctic region (Assing, 2010). According to recent studies, the genus was represented by 11 species in 
Turkey (Assing, 2010; Anlaş et al., 2011), These are Achenium anatolicum Jarrige, 1952; $A$. humile (Nicolai, 1822); A. propontiacum Bordoni, 2009; $A$. scimbalioides Koch, 1937; A. turcicum Coiffait, 1971; A. debile Erichson, 1840; A. caucasicum Laporte,
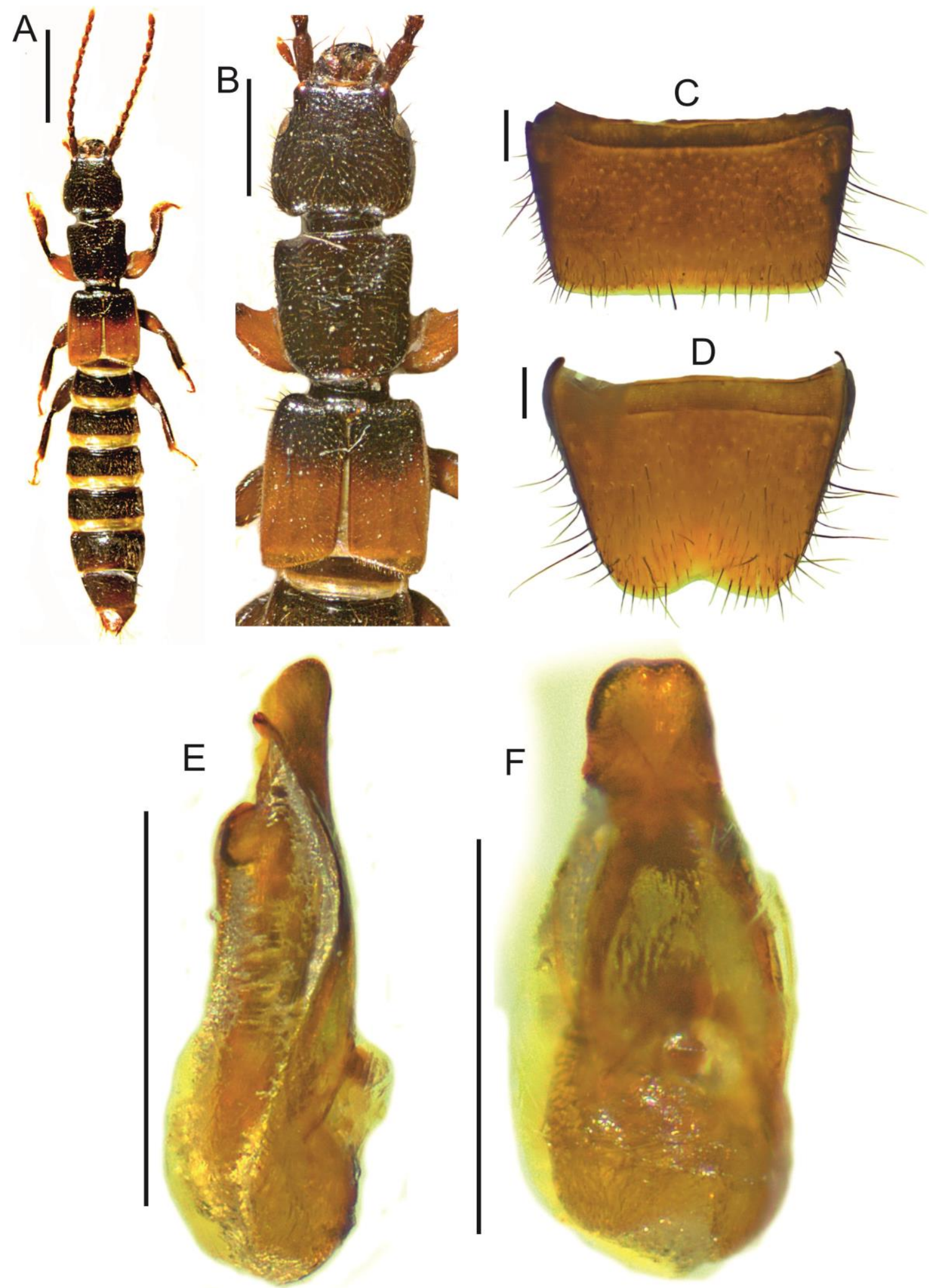

Figure 3. Achenium propontiacum Bordoni. A-habitus; B-forebody; C-male sternite VII; D-male sternite VIII; Eaedeagus, lateral; F-aedeagus, ventral. Scale bars: $1 \mathrm{~mm}$ (A-B); $0.2 \mathrm{~mm}$ (C-D); $0.5 \mathrm{~mm}$ (E-F).

Shekil 3. Achenium propontiacum Bordoni. A-genel vücut; B-ön vücut; $C$-erkek sternit VII; D-erkek sternit VIII; $E$ aedeagus, yan görünüs, F-aedeagus, ön görünüss. Ölçek çubukları: $1 \mathrm{~mm}(A-B) ; 0.2 \mathrm{~mm}(C-D) ; 0.5 \mathrm{~mm}(E-F)$. 

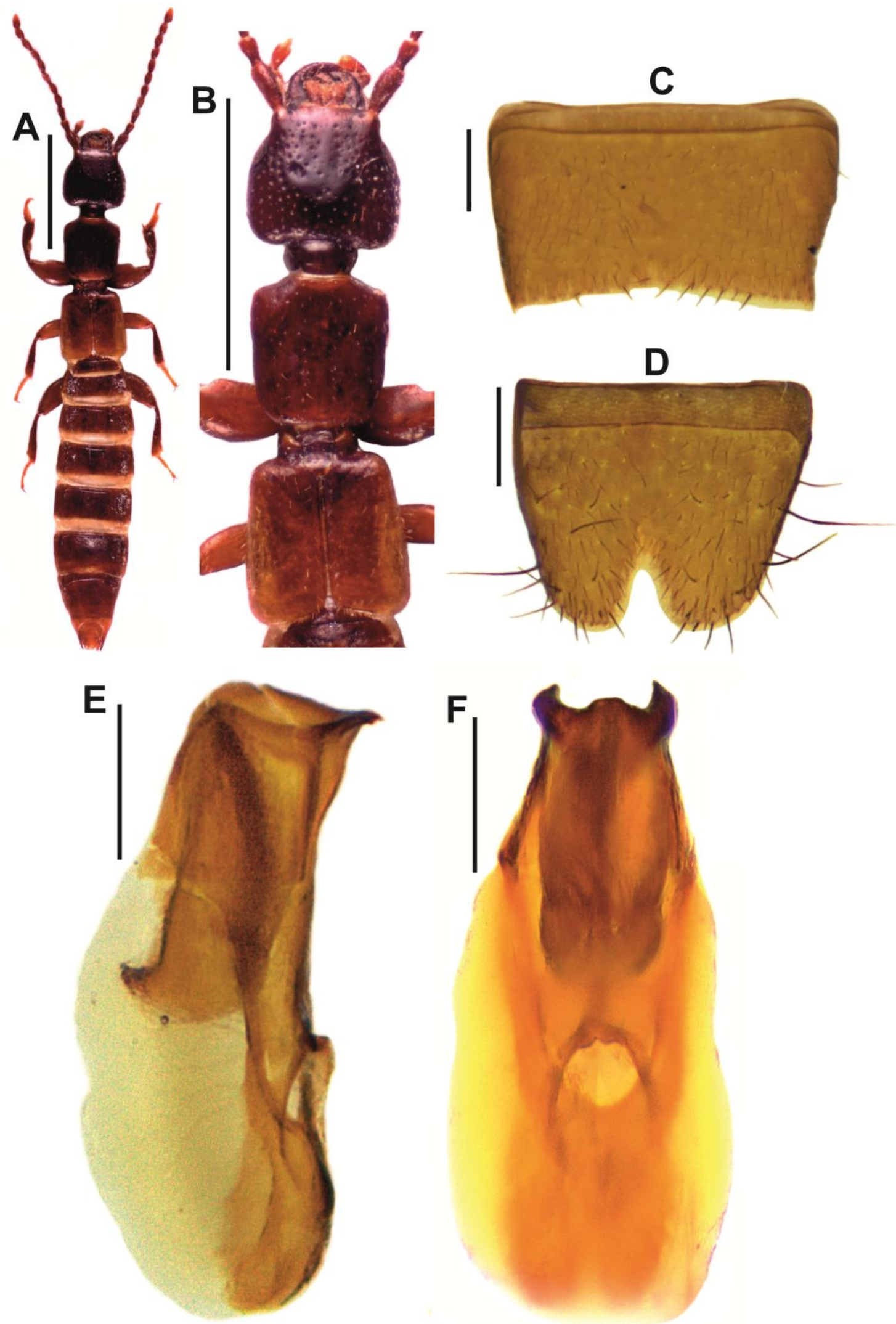

Figure 4. Achenium scimbalioides Koch. A-habitus; B-forebody; C-male sternite VII; D-male sternite VIII; E-aedeagus, lateral; F-aedeagus, ventral. Scale bars: $1 \mathrm{~mm}(\mathrm{~A}-\mathrm{B}) ; 0.2 \mathrm{~mm}(\mathrm{C}-\mathrm{F})$.

Şekil 4. Achenium scimbalioides Koch. A-genel vücut; B-ön vücut; C-erkek sternit VII; D-erkek sternit VIII; Eaedeagus, yan görünüş; F-aedeagus, ön görünüş. Ölçek çubukları: $1 \mathrm{~mm}(A-B) ; 0.2 \mathrm{~mm}(C-F)$. 

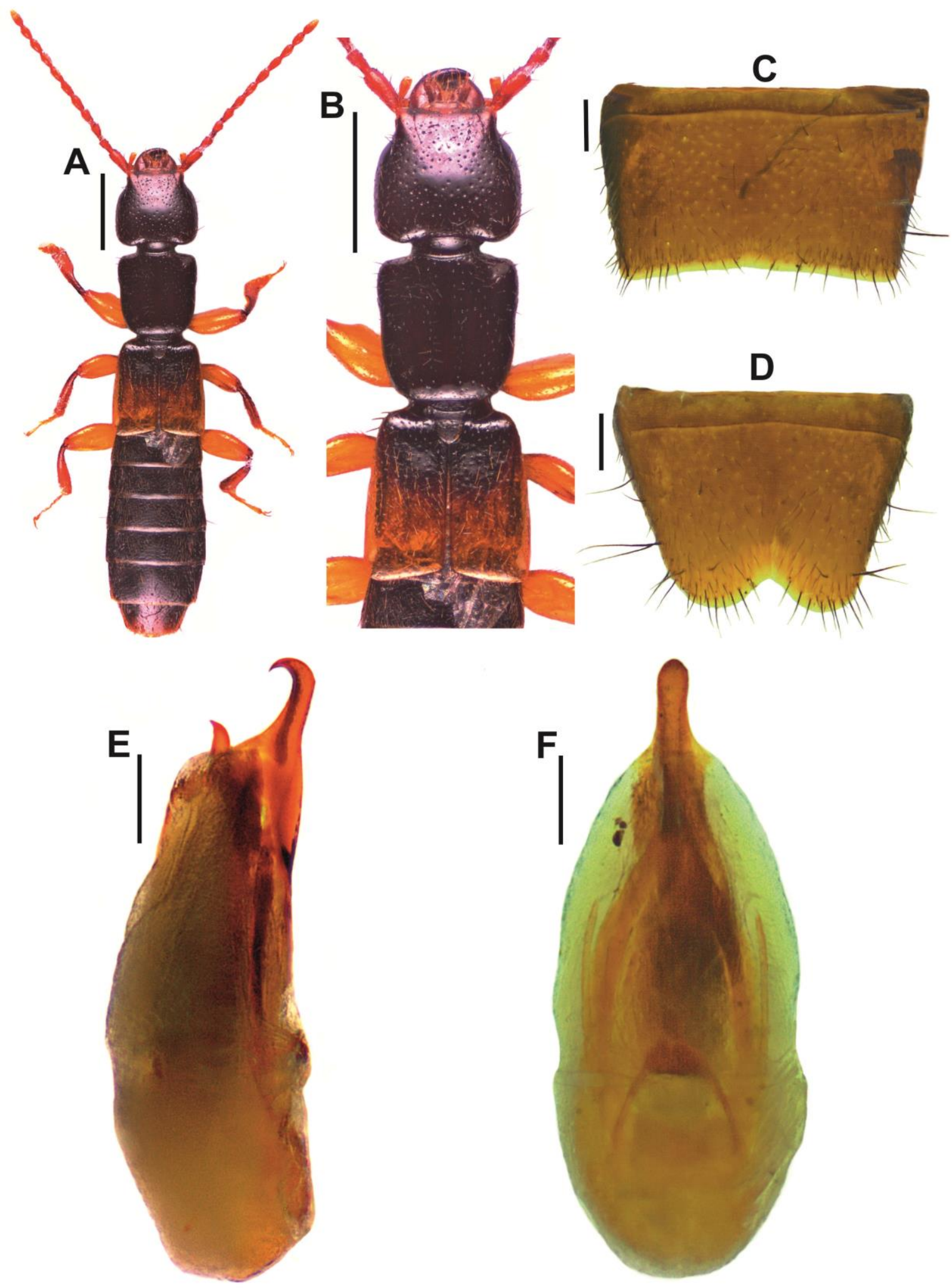

Figure 5. Achenium turcicum Coiffait. A-habitus; B-forebody; C-male sternite VII; D-male sternite VIII; Eaedeagus, lateral; F-aedeagus, ventral. Scale bars: $1 \mathrm{~mm}(\mathrm{~A}-\mathrm{B}) ; 0.2 \mathrm{~mm}(\mathrm{C}-\mathrm{F})$.

Şekil 5. Achenium turcicum Coiffait. A-genel vücut; B-ön vücut; $C$-erkek sternit VII; D-erkek sternit VIII; $E^{-}$ aedeagus, yan görünüs,; F-aedeagus, ön görünüş. Ölçek çubukları: $1 \mathrm{~mm}(A-B) ; 0.2 \mathrm{~mm}(C$-F).

1835; A. picinum Fauvel, 1875; A. quadriceps Eppelsheim, 1889; A.richteri Scheerpeltz, 1961 and $A$. sanctum Koch, 1937. As a result of the field studies in
Aegean and Central Anatolia, a total of five species were recorded: $A$. anatolicum, $A$. humile, $A$. propontiacum, A. scimbalioides and A. turcicum. Of 
the recorded species; $A$. humile from the Aegean Region and A. turcicum from Central Anatolia Region were recorded for the first time. A. turcicum was reported for the first time outside the type locality and its distribution was found to be much wider than previously known. A. debile was reported based on a old specimen from Konya province in central Anatolia region (Assing, 2010). But it was not recorded from central Anatolia in this study. Thus, this record is highly doubtful in central Anatolia.

\section{ACKNOWLEDGEMENTS}

This study was prepared from part of a master thesis approved by the Institute of Natural Sciences of Manisa Celal Bayar University on December 2019. This study was supported by the Scientific and Technological Research Council of Turkey (TÜBİTAK, Project nos: 112T907 and 215Z080).

\section{Statement of Conflict of Interest}

Author has declared no conflict of interest.

\section{Author's Contributions}

The contribution of the authors is equal.

\section{REFERENCES}

Altın Ç, Yağmur E A 2018. Faunistic studies on the species of Paederinae and Xantholinini (Coleoptera: Staphylinidae) in Sarıkamıs forest, Kars province, Turkey. Munis Entomology \& Zoology, 13 (2): 458-462.

Anlaş S 2009. Distributional checklist of the Staphylinidae (Coleoptera) of Turkey, with new and additional records. Linzer biologische Beiträge, 41: 215-342.

Anlaş S, Khachikov E A, Özgen I 2011. On the genus Achenium Leach, 1819 from Turkey and adjacent regions (Coleoptera: Staphylinidae, Paederinae). Zoology in the Middle East, 54: 144-147.
Assing V 2010. A revision of Achenium (Coleoptera: Staphylinidae: Paederinae). Nova Supplementa Entomologica, 21: 1-190.

Assing V 2013. On the Staphylinidae (Coleoptera) of Turkey IX. Five new species, a new synonymy, and additional records. Stuttgarter Beiträge zur Naturkunde A, Neue Serie, 6: 103-125.

Bordoni A 2009. Achenium propontiacum n. sp. della Turchia Europea (Coleoptera, Staphylinidae). Onychium, 7: 53-56.

Coiffait H 1971. Le genre Achenium (Staphylinidae). Nouvelle Revue d'Entomologie, 1: 11-41.

Jarrige J 1952. Brachelytres nouveaux ou mal connus de la faune circamediterraneenne. Annales de la Societe Entomologique de France, 119: 117-139.

Örgel S, Anlaş S 2016. Faunistic studies on the subfamily Paederinae (Coleoptera: Staphylinidae) in Uşak Province, Western Anatolia. Acta Biologica Turcica, 29 (2): 61-66.

Özgen I, Örgel S, Yaman S, Ayaz T 2018. A newly faunistic notes on Staphylinidae (Coleoptera) in Turkey. Munis Entomology \& Zoology, 13 (2): 503506.

Sert O, Turan Y, Şabanoğlu B, Anlaş S, Fırat S 2013. Faunistical, ecological and zoogeographical evaluations on the subfamily Paederinae (Coleoptera: Staphylinidae) in the Central Anatolian Region of Turkey. Turkish Journal of Entomology, 37 (4): 477-492.

Schülke M, Smetana A 2015. Staphylinidae, pp. 3041134. In: I. Löbl, Löbl D. Löbl (Eds.,). Catalogue of Palaearctic Coleoptera. Volume 2. Hydrophiloidea - Staphylinoidea. Revised and updated edition. Leiden: Brill: xxvi, $1702 \mathrm{p}$.

Solodovnikov A, Li X 2013. A new species of the rove beetle genus Achenium from Taman peninsula with the key to the Russian fauna of the genus (Coleoptera: Staphylinidae: Paederinae). Caucasian Entomological Bulletin, 9 (1): 43-46. 